\title{
Postprocedural Thrombosis following Endovascular Treatment of Intracranial Aneurysm with Flow Diverters or Coiling: A Histologic Study
}

\author{
(D).A. Larco, (D) M. Abbasi, (D). Liu, (D) D. Dai, (D) L. Lanzino, (D).E. Savastano, (D) H. Cloft,
(D) D.F. Kallmes, (D). Kadirvel, and (D)W. Brinjikji \\ (D).A. Larco, (D) M. Abbasi, (DY. Liu, (D). Dai, DG. Lanzino, (D).E. Savastano, DH. Cloft,
(D)D.F. Kallmes, DR. Kadirvel, and (D). Brinjikji
}

O- $\equiv$

\begin{abstract}
Endovascular treatment of aneurysms with flow diverters or coiling is sometimes complicated by intraprocedural or postprocedural thrombosis along or within the devices. Thrombus composition and structure associated with such complications may provide insights into mechanisms of thrombus formation and clinical strategies to remove the thrombus. We present a retrospective histopathologic study of 4 patients who underwent mechanical thrombectomy due to acute occlusion of either implanted flow diverter devices or along coils during the treatment of intracranial aneurysm.
\end{abstract}

ABBREVIATIONS: $\mathrm{CitH3}=$ anti-citrullinated $\mathrm{H} 3 ; \mathrm{FD}=$ flow diverter; $\mathrm{GP}=$ glycoprotein; $\mathrm{RBC}=$ red blood cell; $\mathrm{vWF}=$ von Willebrand factor; WBC $=$ white blood cell

$E^{n}$ ndovascular treatment of intracranial aneurysms is becoming more commonplace but is sometimes complicated by in situ thrombus formation along coils in the parent artery or within stents and flow diverters (FDs) due to the thrombogenic nature of these devices. ${ }^{1-3}$ The mechanism of thrombosis is thought to be due to platelet activation because multiple prior studies have shown that complications can be prevented with dual antiplatelet therapy and managed with intra-arterial or intravenous infusion of glycoprotein (Gp) IIb/IIIa inhibitors. When the thrombus is refractory to medical treatment or the distal territory is at risk of infarction, mechanical thrombus removal by endovascular means is performed. Currently, to our knowledge, there are no studies providing insight into the structure of early postprocedural or intraprocedural thrombus related to FDs or coiling procedures. In this study, we sought to evaluate the histopathologic composition and structure of postprocedural or intraprocedural thrombus developing in 4 patients following flow diversion or coiling treatment of aneurysms, with emphasis on the surface characteristics

Received August 16, 2021; accepted after revision October 5.

From the Departments of Radiology (J.A.L., M.A., Y.L., D.D., L.E.S., H.C., D.F.K., R.K. W.B.) and Neurosurgery (J.A.L., M.A., Y.L., G.L., L.E.S., D.F.K., W.B.), Mayo Clinic, Rochester, Minnesota.

J.A. Larco and M. Abbasi contributed equally to this work.

This study is supported by National Institutes of Health grant (No. R01 NS105853) to Waleed Brinjikji and National Institutes of Health grant (No. R01 NS076491) to Ramanathan Kadirvel.

Please address correspondence to Waleed Brinjikji, MD, Departments of Radiology and Neurosurgery, Mayo Clinic, 200 First St SW, Rochester, MN 55905; e-mail: Brinjikji.waleed@mayo.edu; @Wbrinjikji

- Indicates open access to non-subscribers at www.ajnr.org

Indicates article with online supplemental data.

http://dx.doi.org/10.3174/ajnr.A7369 or shell (defined as the outer 10\% of a given thrombus on histologic staining) of these thrombi and implications for therapy.

\section{Case Series}

This was a retrospective histopathologic study of 4 patients who underwent mechanical thrombectomy due to acute occlusion of either implanted FD devices or along coils during the treatment of an intracranial aneurysm. This study was approved by the Mayo Clinic institutional review board; the need for informed consent was waived. All embolizations were performed with the patient under full anticoagulation with an activated clotting time of 250300 seconds. FD treatments were performed after loading patients with aspirin and clopidogrel for elective cases or infusion of tirofiban for acute cases. Mechanical thrombectomy was performed by members of our neurointerventional team using $5 \mathrm{~F}$ and $6 \mathrm{~F}$ catheters (Sofia; MicroVention) using the direct aspiration technique.

\section{Patient 1}

A 37-year-old woman with a medical history of migraines, tobacco use, and a family history of intracranial aneurysms underwent elective FD placement for a 12-mm left, supraclinoid ICA aneurysm diagnosed during screening, given her family history of intracranial aneurysms. She was placed on $325 \mathrm{mg}$ of aspirin and $75 \mathrm{mg}$ of clopidogrel for 5 days preoperatively, and no platelet aggregation testing was performed. The FD deployment was complicated by stent migration that was found to be partially covering the left anterior cerebral artery (Fig 1), and a second stent was deployed. Five days later, she presented to the emergency department after suddenly developing right-sided hemiparesis and aphasia with an NIHSS score of 9. Noncontrast CT demonstrated no evidence of acute 


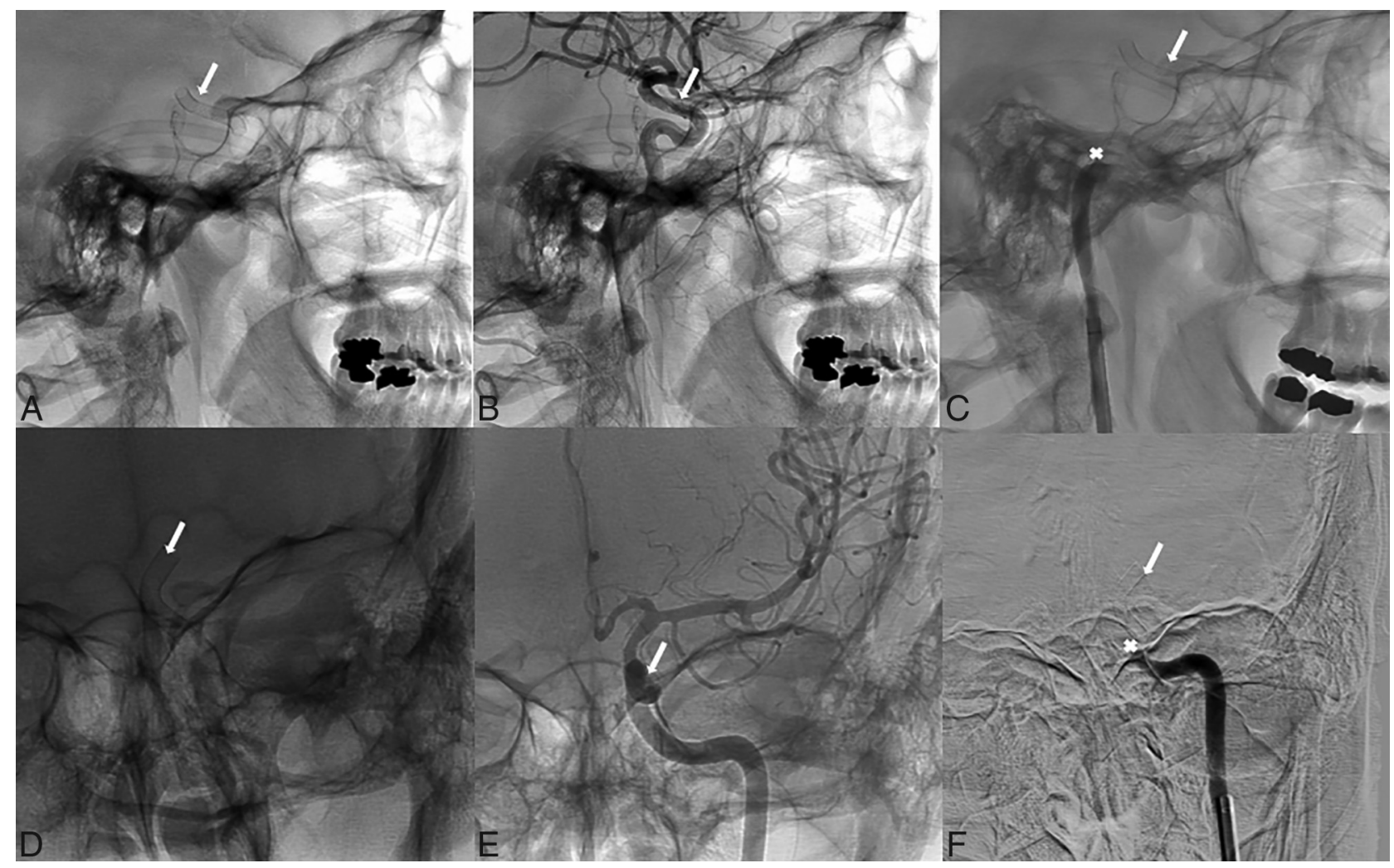

FIG 1. Lateral view showing the final positioning of the FD (white arrow) $(A)$, with a post-procedural contrast-enhanced angiogram (B) and the occlusion site that involves the carotid artery (white asterisk) (C). Anterior-posterior view shows the final position of the FD (D), with a postprocedural contrast-enhanced angiogram $(E)$ and the occlusion site that involves the carotid artery $(F)$.

ischemic stroke or intracranial bleeding. She underwent cerebral angiography, which confirmed the presence of acute stent thrombosis, which was treated with mechanical thrombectomy using a $6 \mathrm{~F}$ catheter (Sofia Plus; MicroVention), resulting in successful recanalization (modified TICI $2 \mathrm{~b}$ ) with 1 pass using thromboaspiration. A continuous tirofiban infusion was started for the patient, and follow-up angiography was performed to confirm the patency of the stent. The retrieved thrombus was sent for histologic evaluation. The patient was discharged with no functional disability, and we prescribed dual antiplatelet therapy, switching her from clopidogrel to ticagrelor.

As depicted in Fig 2, the retrieved thrombus was predominantly composed of red blood cells (RBCs) $(78.9 \%)$ followed by fibrin (13.2\%), platelets (4.4\%), and white blood cells (WBCs) (3.4\%). On immunohistochemistry, the average percentages of platelets (CD42b), von Willebrand factor (vWF), and anti-citrullinated H3 (CitH3, a marker of neutrophil extracellular traps) were $26.1 \%$, $23.7 \%$, and $5.6 \%$, respectively. Additionally, thrombus showed high expression of platelets (CD42b) (67.07\%), vWF (34.3\%), and CitH3 (28.6\%) in the shell of the thrombus (Online Supplementary Data). Inside the thrombus, there were multiple platelet islands surrounded by thick fibrin strains. Within the thrombus, there were RBC-rich portions, which were associated with thin fibrin strains and low expression of CitH3 and vWF (Fig 2).

\section{Patient 2}

A 90-year-old woman with a medical history of atrial fibrillation, non-ST-elevation myocardial infarction hypertension, peripheral artery disease, gastrointestinal bleeding, and subdural hemorrhage presented to our institution with acute headache, rightsided ptosis, myosis, and intractable vomiting. CT and CTA on admission demonstrated a large subarachnoid hemorrhage secondary to bleeding from a posterior communicating artery aneurysm along with intraventricular extension. Cerebral angiography demonstrated a $6.5 \times 8 \mathrm{~mm}$ wide-neck right posterior communicating artery aneurysm with an adjacent daughter sac along with fusiform dilation of the supraclinoid ICA just distal to the ruptured aneurysm. The patient was treated with a FD (Pipeline Embolization Device, $4.5 \times 18 \mathrm{~mm}$, PED; Medtronic) and coil embolization $(8 \times 20$ Axium $3 \mathrm{D}$ coil and a $4 \times 12$ Axium helical coil; Covidien) after attempted balloon-assisted coiling. A control angiogram demonstrated patency of the stent and intra-aneurysmal thrombosis (Fig 3). The patient was immediately started on a tirofiban infusion. However, immediately after the procedure, she developed left-sided hemiparesis. A new CT scan revealed an early right-hemispheric cerebral infarction secondary to in-stent thrombosis for which she underwent mechanical thrombectomy using a 6F catheter (Sofia Plus) with successful recanalization.

The retrieved thrombus was sent for histologic evaluation. Unfortunately, she had a large, right-hemispheric stroke and died 3 days later because the family opted for comfort measures only. Histomorphology of the thrombus retrieved from this patient is depicted in Fig 4. The thrombus was mainly made of RBCs (52.6\%) followed by fibrin (26.9\%), platelets (17.1\%), and WBCs (3.2\%). The average percentages of platelets (CD42b), vWF, and 

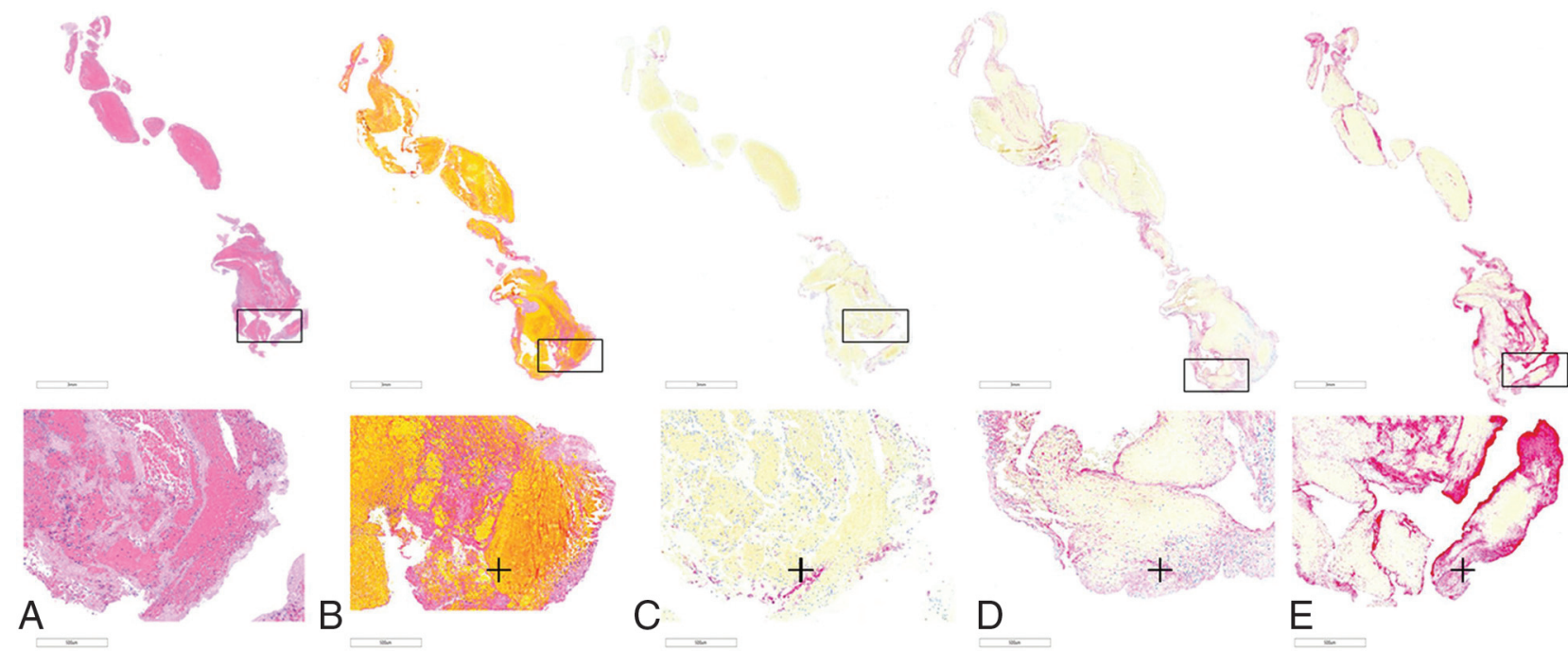

FIG 2. Staining of the thrombus retrieved from patient 1 showing the structure of the thrombus on H\&E staining $(A)$, with a high percentage of RBCs in the thrombus ( $B$, Martius Scarlet Blue, stained yellow, identified with the plus sign). Peripheral expression of neutrophil extracellular traps (CitH3) (C, stained red, identified with the plus sign), vWF (D, stained pink and red, identified with the plus sign), and platelets (CD42b) (E, stained red, identified with the plus sign) in the thrombus.

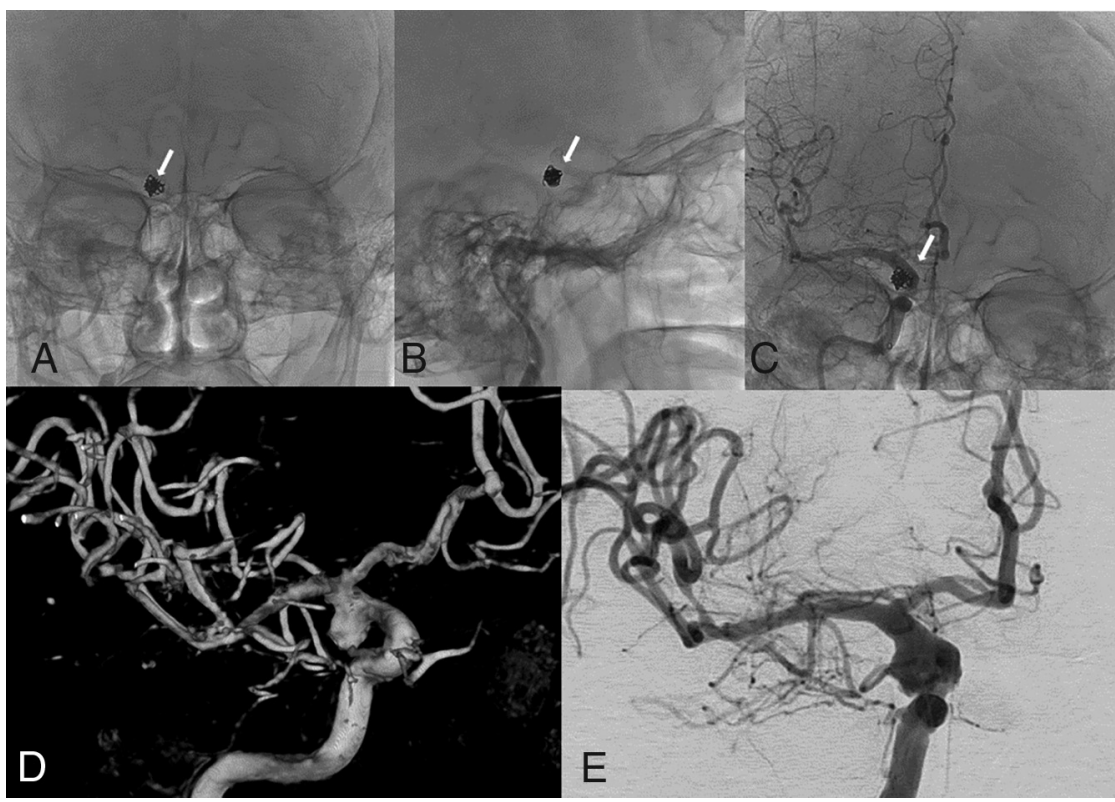

FIG 3. FD implantation with coiling $(A-E)$. Anterior-posterior $(A)$ and lateral $(B)$ views of the postprocedural positioning of the coiled aneurysm (white arrows). Anterior-posterior contrastenhanced angiogram shows postprocedural patency of the vasculature $(C)$. 3D reconstruction and a contrast-enhanced angiogram show the vasculature architecture and a wide-neck right posterior commuting artery aneurysm with an adjacent daughter sac $(D$ and $E)$.

CT on admission demonstrated a large subarachnoid hemorrhage with ventricular extension complicated by obstructive hydrocephalus. The patient became comatose acutely, requiring intubation and the placement of an external intraventricular drain. Cerebral angiography demonstrated an $8.4 \times 6.8 \times 6.6 \mathrm{~mm}$ lobulated anterior communicating artery aneurysm, which was treated with coil embolization $(6 \times 15 \mathrm{~mm}$ complex Axium, $5 \times 10 \mathrm{~mm}$ helical Axium, $4 \times$ $8 \mathrm{~mm}$ helical Axium, $3 \times 6 \mathrm{~mm}$ helical Axium coils). The final control angiogram demonstrated occlusion of the right MCA, which was recanalized in 1 pass of the thrombectomy device (5F catheter, Sofia, Fig 5).

The retrieved thrombus was sent for histologic evaluation. During the ensuing 2 days, the patient's neurologic condition did not improve, and he died 7 days after the initial procedure. Similar to the first 2 cases of flow diversion, the thrombus was mainly composed of RBCs (65.3\%) followed by

CitH3 were 31.2\%, 29.1\%, and 6.4\%, respectively (Fig 4). Similar to the first case, this thrombus also had high expression of platelets (CD42b) (78.1\%), vWF (73.4\%), and CitH3 (28.3\%) in the shell of the thrombus (Online Supplementary Data).

\section{Patient 3}

A 75-year-old man with a history of gastroesophageal reflux disease and hyperlipidemia, in treatment for colon cancer, presented to our institution with headache, aphasia, dizziness, and confusion. Head fibrin (17.6\%), platelets (13.1\%), and WBCs (4\%). The average percentages of platelets (CD42b), vWF, and CitH3 were 37.8\%, 11.2\%, and $16.4 \%$, respectively (Fig 6). This thrombus also showed high expression of platelets (CD42b) (61.5\%), vWF (18.3\%), and CitH3 $(20.2 \%)$ in the shell of the thrombus (Online Supplemental Data).

\section{Patient 4}

A 29-year-old woman with unremarkable medical history or previous hospitalizations presented to our institution with a 

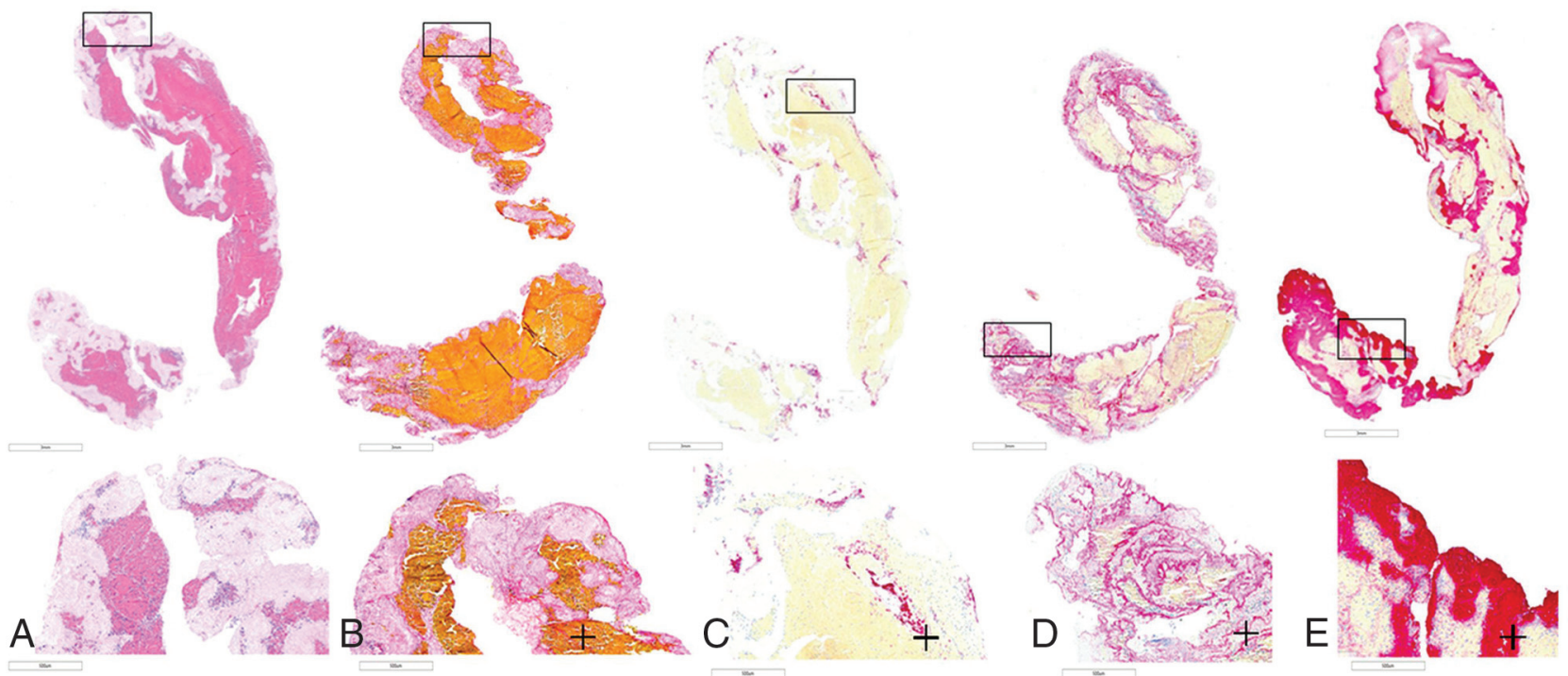

A.
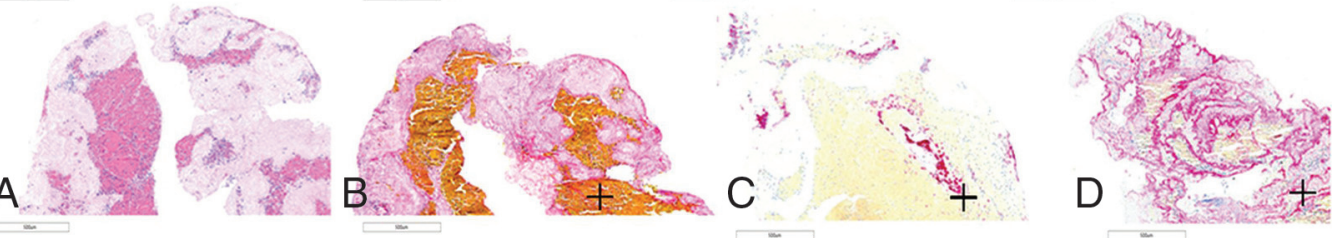

FIG 4. Staining of the thrombus retrieved from patient 2 showing the structure of the thrombus on H\&E staining $(A)$ with a high percentage of RBCs in the thrombus ( $B$, Martius Scarlet Blue, stained yellow, identified with the plus sign). Peripheral expression of neutrophil extracellular traps (CitH3) (C, stained red, identified with the plus sign), vWF (D, stained pink and red, identified with the plus sign), and platelets (CD42b) (E, stained red, identified with the plus sign) in the thrombus.

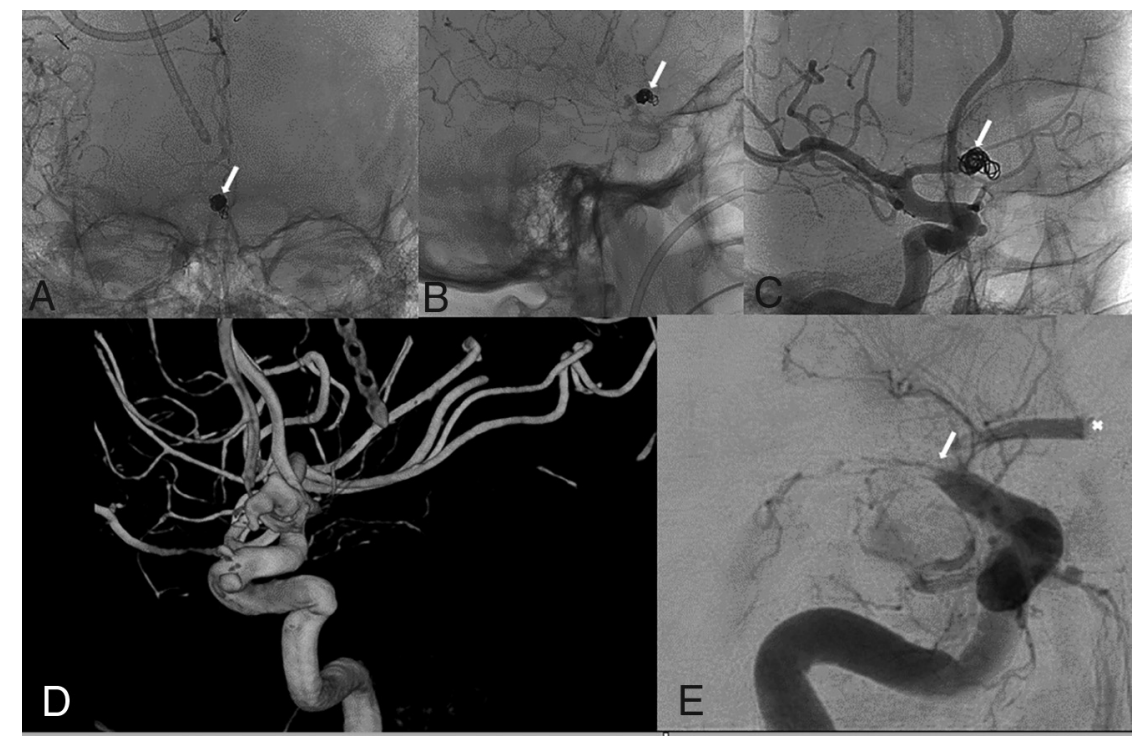

FIG 5. Anterior-posterior $(A)$ and lateral $(B)$ views of the postprocedural positioning of the coiled aneurysm (white arrows). Lateral projection of a contrast-enhanced angiogram and the external intraventricular drain shows in the background (C). 3D reconstruction shows the vascular architecture neighboring the lobulated anterior communicating artery aneurysm $(D)$. Anteroposterior DSA projection demonstrates acute large vessel occlusion (E) (white asterisk).

of a $5 \mathrm{~F}$ catheter (Sofia) were required to recanalize the artery. The retrieved thrombus was sent for histologic evaluation. The patient made a full recovery. As depicted in Fig 8, thrombus retrieved from this patient was composed of RBCs (36.8\%) followed by fibrin (36.3\%), platelets $(24.8 \%)$, and WBCs (2.1\%). On immunohistochemistry, the average percentages of platelets (CD42b), vWF, and CitH3 were 46.1\%, $12.3 \%$, and $17.2 \%$, respectively (Fig 8 ). Additionally, thrombus showed high expression of platelets (CD42b) (71.6\%), vWF (36.7\%), and $\mathrm{CitH} 3$ (35.1\%) in the shell of the thrombus. Thrombus had multiple platelet islands surrounded by thick fibrin strains. RBC-rich portions were correlated with thin fibrin strains and low expression of $\mathrm{CitH} 3$ and vWF (Online Supplemental Data).

All retrieved thrombi were predominantly composed of RBCs, with an average of $58.2 \%$. The average percentages of platelets (CD42b), vWF,

thunderclap headache radiating to the neck and associated with nausea and vomiting. Noncontrast head CT demonstrated a diffuse subarachnoid hemorrhage in an aneurysmal pattern with a mild intraventricular hemorrhage. A left ICA cerebral angiogram demonstrated a $4 \times 4.5 \times 4.2 \mathrm{~mm}$ lobulated aneurysm at the origin of the posterior communicating artery, which was treated with coil embolization (2.5-mm 3D, $1.5-\mathrm{mm}$ helical coils, Axium, Fig 7). The procedure was complicated by an embolus to the left M2 trunk that was treated with aspiration thrombectomy. Two passes and $\mathrm{CitH} 3$ were $35.3 \%, 19.1 \%$, and $11.4 \%$, respectively. Additionally, all thrombi showed high expression of platelets (CD42b) (69.6\%), vWF (40.7\%), and CitH3 (29.6\%) in the shell.

\section{DISCUSSION}

Postprocedural thrombosis is a common complication following intracranial aneurysm treatment with FDs or coiling. In the present study, we performed histologic evaluation of thrombi retrieved from 4 patients with this complication and were able to provide 

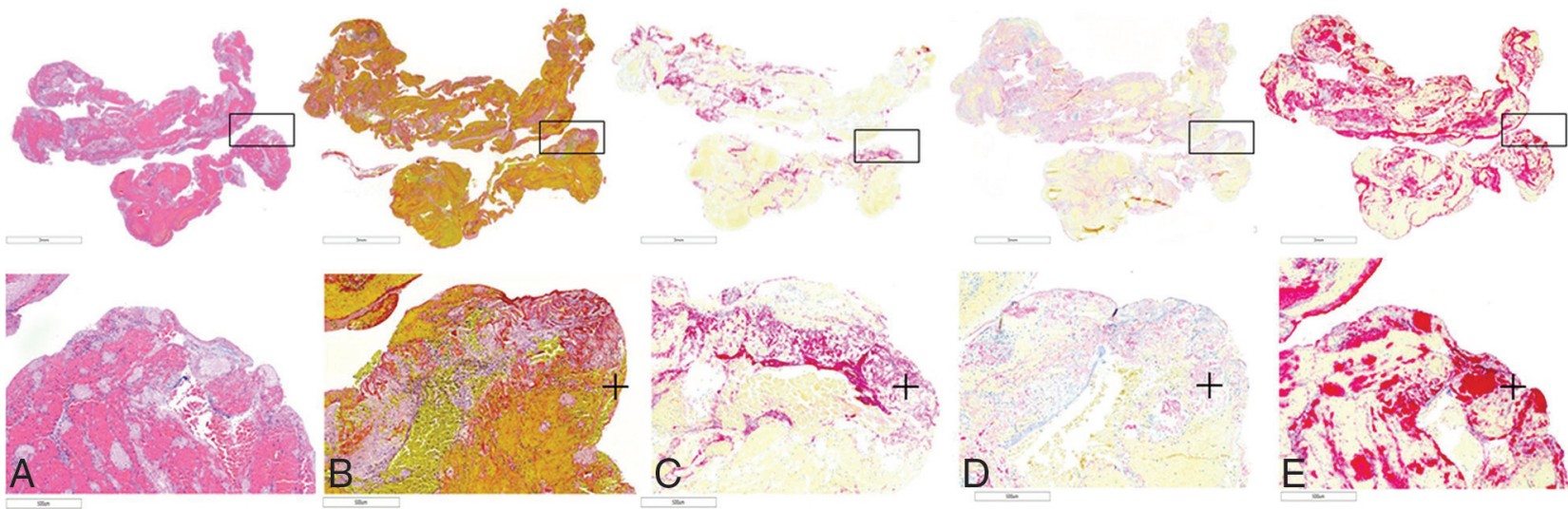

FIG 6. Staining of the thrombus retrieved from patient 3 showing the structure of thrombus on H\&E staining $(A)$ with a high percentage of RBCs in the thrombus ( $B$, Martius Scarlet Blue, stained yellow, identified with the plus sign). Peripheral expression of neutrophil extracellular traps (CitH3) (C, stained red, identified with the plus sign), vWF (D, stained pink and red, identified with the plus sign), and platelets (CD42b) (E, stained red, identified with the plus sign) in the thrombus.

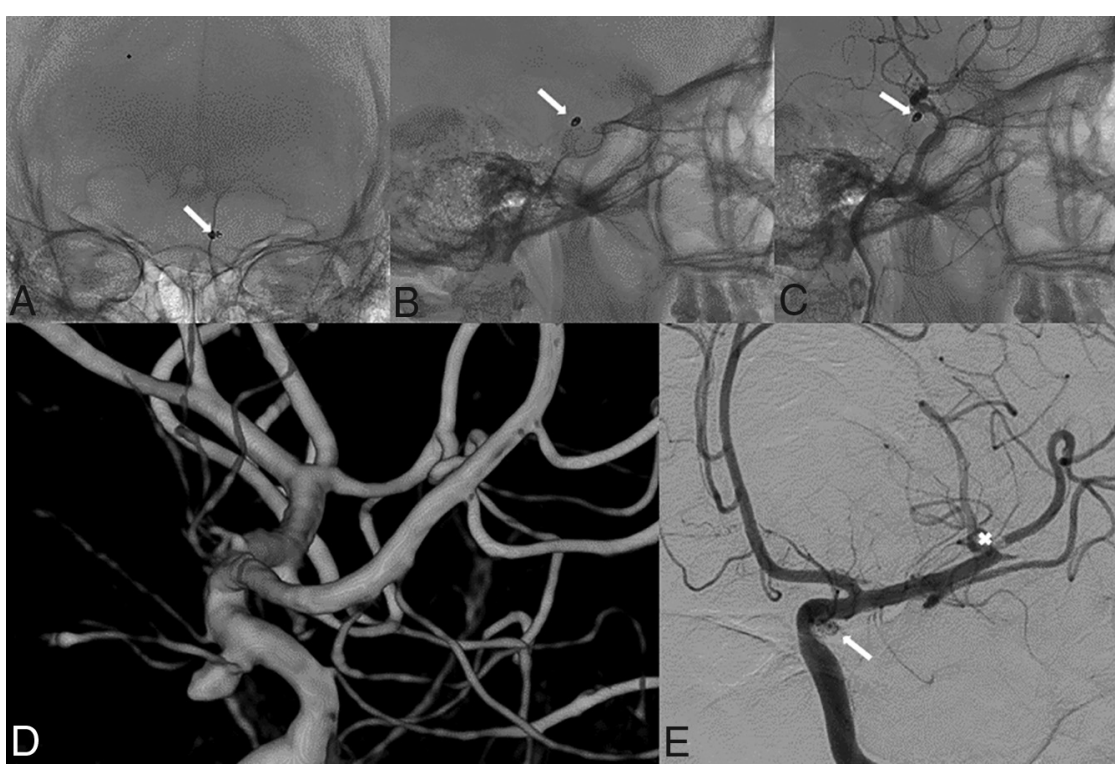

FIG 7. Anterior-posterior $(A)$ and lateral $(B)$ views of the postprocedural positioning of the coiled aneurysm (white arrows) and the lateral projection of contrast-enhanced DSA (C) showing patency of the artery. 3D reconstruction $(D)$ of the lobulated aneurysm located in the posterior communicating artery. Anterior-posterior DSA (E) projection shows occlusion of M2 (white asterisk).
A number of variables have been reported as contributory to in situ thrombus formation within FDs. These include the thrombogenic character of materials used to construct FDs, malapposition or movement of FDs, and resistance to antiplatelet agents. ${ }^{3}$ Even third-generation FDs with surface modifications with synthetic biocompatible phosphorylcholine seem to have similar outcomes compared with previous generations of FDs. $^{5-7}$

Noncompliance with dual antiplatelet therapy and resistance to clopidogrel therapy are also known causes of in-stent thrombosis; however, there is controversy regarding the use of platelet function testing prior to FD implantation and converting known nonresponders to other antiplatelet medications such as ticagrelor or prasugrel. ${ }^{8}$ Although certainly attention to patient compliance and identifying clopidogrel nonresponders before FD treatment should reduce the incidence some unique insights into the mechanisms of thrombosis and clot characteristics in these situations. We found that these thrombi are mostly rich in RBCs but express high levels of platelets, vWF, and Cit-H3 on the periphery and surface, resulting in formation of shell or shell-like encapsulation around the thrombus. This unique structure of these thrombi explains the resistance to IV thrombolytics as demonstrated by Staessens et al. ${ }^{4}$ The distinct pattern of in situ thrombus in patients treated with FDs may be due to a combination of factors, including endothelial injury during embolization and the dense mesh metal in the FDs and the platinum of embolization coils being thrombogenic in nature.

The formation of thrombus in FDs could be due to FD-related factors or patient-related factors. of thrombosis formation, we would still have some patients who may have this complication regardless of clopidogrel responsiveness and compliance with medications. One explanation could be related to diurnal fluctuations in platelet reactivity. One study has suggested that clopidogrel activity could vary during the day with peak platelet reactivity in the early morning. ${ }^{9}$

A number of techniques have been reported for revascularization of thromboembolic events during coil embolization or FD treatment. ${ }^{10}$ In a prior era, intravascular fibrinolysis with intraarterial or even IV tPA was used. However, due to decreased efficacy and increased knowledge from the cardiology literature, many interventionalists began administering Gp IIb/IIIa inhibitors such as abciximab and tirofiban with improved results. ${ }^{11}$ In a 


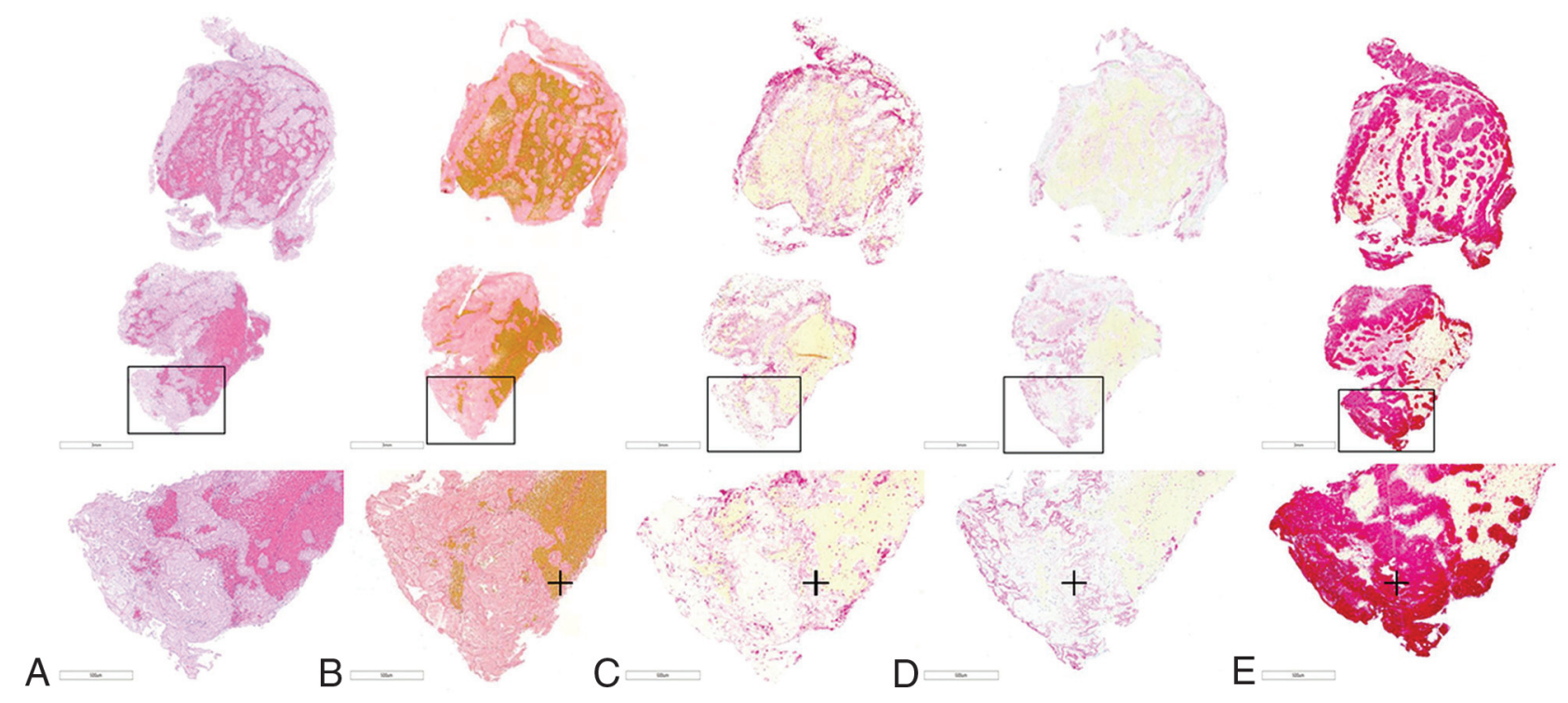

FIG 8. Staining of the thrombus retrieved from patient 4 showing the structure of thrombus on H\&E staining $(A)$, with a high percentage of RBCs in the thrombus ( $B$, Martius Scarlet Blue, stained yellow, identified with the plus sign). Peripheral expression of neutrophil extracellular traps $(\mathrm{CitH} 3)(C$, stained red, identified with the plus sign), vWF (D, stained pink and red, identified with the plus sign), and platelets (CD42b) (E, stained red, identified with the plus sign) in the thrombus.

study of 254 patients with cerebral aneurysms undergoing rescue therapy during aneurysm embolization, it was found that patients who received Gp IIb/IIIa inhibitors had significantly lower rates of mortality and morbidity compared with those receiving tPA. ${ }^{12}$ Study by Delvoye et $\mathrm{al}^{13}$ accentuated the essential role that GP IIb/ IIIa inhibitors play during acute thromboembolic events following endovascular treatment for intracranial aneurysms. The authors compared the in vitro/in vivo effects on thrombus formation of abciximab versus alteplase. Abciximab resulted in a higher rate of final recanalization without increasing the risk of hemorrhage. In a meta-analysis of 23 studies and 516 patients, it was reported that the rate of morbidity was significantly lower in patients treated with Gp IIb/IIIa inhibitors (11\% versus 29\%) and recanalization rates were significantly higher ( $72 \%$ versus $50 \%)$. Notably, there was no difference in intra-arterial-versus-IV administration of Gp IIb/IIIa inhibitor efficacy or safety. ${ }^{14}$

Empirically, it seems that the neurointerventional community has realized that platelets are the key to the formation of these in situ thrombi. However, to date, little is known regarding the composition of these thrombi in actual patients. Our study confirmed the importance of platelets as the mediator of thrombosis because our cases had a shell of platelets, vWF, and neutrophil extracellular traps in the periphery of the clot. ${ }^{4}$ As described for each case, we have observed the difference in fibrin structure between platelet-rich areas and RBC-rich areas. Within platelet-rich areas, we observed a thick fibrin strain, whereas RBC-rich areas were associated with thin fibrin strains (Online Supplemental Data). This finding explains the efficacy of Gp IIb/IIIa inhibitors in these patients and why IV tPA fails. In fact, recently published a study examining characteristics of thrombolysis-refractory clots have reported that a high proportion of these have a fibrinolytic-resistant shell consisting of platelets and vWF, similar to findings in our study. ${ }^{15}$ The high expression of vWF in the shell of these retrieved thrombi may suggest that some degree of endothelial damage must have precipitated the periprocedural thrombosis as well as that this primarily comes from the subendothelial connective tissues.

There are limitations to this study. First, only 4 patients were included. Second, we were not able to compare the different dual antiplatelet therapies for preventing thrombosis formation and their effect on clot structure. Third, we were also not able to compare bare or coated FDs or coils in terms of thrombus composition or structure because only PEDs or bare platinum coils were used in our cases. Another limitation of the study is that it was a retrospective single-center study and it may not be generalizable to other centers.

\section{CONCLUSIONS}

Postprocedural thrombosis following endovascular treatment of aneurysms is associated with high expression of neutrophil extracellular traps, platelets, and vWF on the thrombus periphery, explaining why these thrombi can be resolved far better with Gp IIb/IIIa inhibitors than with fibrinolytics. More histologic studies are needed to better understand the structure and composition of these types of thrombosis and their association with the timing of thrombosis formation and prevention strategies.

Disclosure forms provided by the authors are available with the full text and PDF of this article at www.ajnr.org.

\section{REFERENCES}

1. Guglielmi G, Vinuela F, Sepetka I, et al. Electrothrombosis of saccular aneurysms via endovascular approach, Part 1: electrochemical basis, technique, and experimental results. J Neurosurg 1991;75:1-7 CrossRef Medline

2. Cognard C, Pierot L, Anxionnat R, et al; Clarity Study Group. Results of embolization used as the first treatment choice in a consecutive nonselected population of ruptured aneurysms: clinical results of the Clarity GDC study. Neurosurgery 2011;69:837-41 CrossRef Medline 
3. Townsend RK, Wolfe SQ, Anadani M, et al. Endovascular management of acute postprocedural flow diverting stent thrombosis. $J$ Neurointerv Surg 2020;12:67-71 CrossRef Medline

4. Staessens S, Denorme F, François O, et al. Structural analysis of ischemic stroke thrombi: Histological indications for therapy resistance. Haematologica 2020;105:498-507 CrossRef Medline

5. Atasoy D, Kandasamy N, Hart J, et al. Outcome study of the Pipeline embolization device with shield technology in unruptured aneurysms (PEDSU). AJNR Am J Neuroradiol 2019;40:2094-2101 CrossRef Medline

6. Martinez-Galdamez M, Lamin SM, Lagios KG, et al. Treatment of intracranial aneurysms using the Pipeline Flex embolization device with shield technology: angiographic and safety outcomes at 1year follow-up. J Neurointerv Surg 2019;11:396-99 CrossRef Medline

7. Rice H, Galdámez MM, Holtmannspötter M, et al. Periprocedural to 1year safety and efficacy outcomes with the Pipeline Embolization Device with Shield technology for intracranial aneurysms: a prospective, post-market, multi-center study. J Neurointerv Surg 2020;12:110712 CrossRef Medline

8. Gupta R, Moore JM, Griessenauer CJ, et al. Assessment of dual-antiplatelet regimen for Pipeline embolization device placement: a survey of major academic neurovascular centers in the United States. World Neurosurg 2016;96:285-92 CrossRef Medline

9. Freynhofer MK, Hein-Rothweiler R, Haller PM, et al. Diurnal variability of on-treatment platelet reactivity in clopidogrel versus prasugrel treated acute coronary syndrome patients: a pre-specified TROPICAL-ACS Sub-Study. Thromb Haemost 2019;119:66067 CrossRef Medline

10. Park KY, Yeon JY, Kim BM, et al. Efficacy and safety of flow-diverter therapy for recurrent aneurysms after stent-assisted coiling. AJNR Am J Neuroradiol 2020;41:663-68 CrossRef Medline

11. Jeong HW, Jin SC. Intra-arterial infusion of a glycoprotein IIb/IIIa antagonist for the treatment of thromboembolism during coil embolization of intracranial aneurysm: a comparison of abciximab and tirofiban. AJNR Am J Neuroradiol 2013;34:1621-25 CrossRef Medline

12. Brinjikji W, McDonald JS, Kallmes DF, et al. Rescue treatment of thromboembolic complications during endovascular treatment of cerebral aneurysms. Stroke 2013;44:1343-47 CrossRef Medline

13. Delvoye F, Loyau S, Labreuche J, et al. Intravenous abciximab as a rescue therapy for immediate reocclusion after successful mechanical thrombectomy in acute ischemic stroke patients. Platelets 2021 April 11. [Epub ahead of print] CrossRef Medline

14. Brinjikji W, Morales-Valero SF, Murad MH, et al. Rescue treatment of thromboembolic complications during endovascular treatment of cerebral aneurysms: a meta-analysis. AJNR Am J Neuroradiol 2015;36:121-25 CrossRef Medline

15. Di Meglio L, Desilles JP, Ollivier V, et al. Acute ischemic stroke thrombi have an outer shell that impairs fibrinolysis. Neurology 2019;93:E1686-98 CrossRef Medline 